\title{
Self-sampling for community respiratory illness: a new tool for national virological surveillance
}

A J Elliot (Alex.Elliot@phe.gov.uk)1, A Bermingham², A Charlett², A Lackenby², J Ellis², C Sadler², P Sebastianpillai², C Powers², D Foord ${ }^{3}$, E Povey ${ }^{3}$, B Evans 2 , H Durnall ${ }^{4}$, D M Fleming ${ }^{4}$, D Brown ${ }^{2}$, G E Smith ${ }^{1}$, M Zambon ${ }^{2}$

1. Public Health England, Birmingham, United Kingdom

2. Public Health England, London, United Kingdom

3. National Health Service Direct, Milton Keynes, United Kingdom

4. Royal College of General Practitioners Research and Surveillance Centre, Birmingham, United Kingdom

Citation style for this article:

Elliot AJ, Bermingham A, Charlett A, Lackenby A, Ellis J, Sadler C, Sebastianpillai P, Powers C, Foord D, Povey E, Evans B, Durnall H, Fleming DM, Brown D, Smith GE, Zambon M. Self-sampling for community respiratory illness: a new tool for national virological surveillance. Euro Surveill. 2015;20(10):pii=21058. Available online: http://www.eurosurveillance.org/ViewArticle.aspx?Articleld $=21058$

Article submitted on 03 September 2013/ published on 12 March 2015

This report aims to evaluate the usefulness of selfsampling as an approach for future national surveillance of emerging respiratory infections by comparing virological data from two parallel surveillance schemes in England. Nasal swabs were obtained via self-administered sampling from consenting adults ( $\geq 16$ yearsold) with influenza symptoms who had contacted the National Pandemic Flu Service (NPFS) health line during the 2009 influenza pandemic. Equivalent samples submitted by sentinel general practitioners participating in the national influenza surveillance scheme run jointly by the Royal College of General Practitioners (RCGP) and Health Protection Agency were also obtained. When comparable samples were analysed there was no significant difference in results obtained from self-sampling and clinician-led sampling schemes. These results demonstrate that selfsampling can be applied in a responsive and flexible manner, to supplement sentinel clinician-based sampling, to achieve a wide spread and geographically representative way of assessing community transmission of a known organism.

\section{Introduction}

The $2009 \mathrm{~A}\left(\mathrm{H}_{1} \mathrm{~N}_{1}\right)$ influenza global pandemic presented major challenges for health systems around the world in both developed and resource limited countries. Accurate recognition of viral transmission in the community and predictive assessment of trends in clinical morbidity were required to optimise specific interventions such as antiviral prophylaxis and vaccination of risk groups and more general social distancing measures such as school closures. Novel and flexible approaches to surveillance were required during these periods of rapidly changing disease indicators and fluctuating demand for healthcare delivery.

During the initial phase of the pandemic in England (May to June 2009), laboratory testing focused on patients who fulfilled the national algorithm [1]. The case definition targeted travellers returning to the
United Kingdom (UK) from high risk countries (e.g. Mexico) presenting for treatment, mainly in secondary care settings. In parallel, general practitioner (GP) virological surveillance was enhanced to provide an estimate of community morbidity due to the newly emerged influenza $A\left(\mathrm{H}_{1} \mathrm{~N}_{1}\right)$ pdmog virus. Aggregation of data from laboratory-confirmed cases provided a reasonable estimate of growth and spread of the pandemic but as the pandemic progressed, the pattern of healthcare provision shifted.

Rather than contact GPs or visit emergency departments, patients were encouraged to use the national telephone helpline (NHS Direct (NHSD); note that NHSD ceased operations on 31 March 2014). In July 2009, amid the first wave of the pandemic, an influenza-specific telephone and web-based health service was launched (National Pandemic Flu Service (NPFS)) that authorised the collection of oseltamivir for those patients over the age of one year, with no respiratory complications and with no underlying medical conditions who were suspected of having pandemic influenza $A\left(\mathrm{H}_{1} \mathrm{~N}_{1}\right)$. NPFS continued through the second wave of the pandemic until early in 2010. Virological surveillance of those in the community seeking access to medical care through these alternative routes was undertaken through selfsampling. The feasibility of this approach had been previously demonstrated during seasonal influenza [2] and was instigated during May 2009 to assist the provision of accurate estimates of number of cases [3].

Here, virological surveillance data, including semiquantitative analysis of viral load, obtained from patient self-sampling is compared with GP (clinician) sampling to assess the usefulness of this approach for future national surveillance of emerging respiratory infections. 


\section{Methods}

\section{Community self-sampling}

Self-sampling was based upon previously validated methodologies for both logistics and laboratory analyses $[2,3]$. In brief, symptomatic members of the public who had used either the national telephone service (NHSD or NPFS) and/or the website interface, who were symptomatic for 'cold/influenza' symptoms and issued a prescription/voucher for oseltamivir, were selected for the self-sampling scheme. Each day, equal numbers of eligible participants in England were randomly selected per region based on Strategic Health Authority (SHA) boundaries. A sampling kit similar to those provided to sentinel GPs but modified to fit through a standard letterbox, was sent to the participant's home address. Each kit included a personalised introductory letter, a patient information leaflet explaining the scheme, an instructional sheet on how to take a nasal swab sample, a dry swab, a vial of virus transport medium (VTM) and a short epidemiological questionnaire requesting information on basic patient demographics (age/sex), presenting symptoms and date of onset, date of swabbing and antiviral treatment (start date and number of doses of antivirals, if taken). Pre-paid packaging (which complied with the UN 3373 regulation) was also provided with instructions to return specimens by the postal system (at no cost to the patient) to the Health Protection Agency (HPA; the HPA became part of Public Health England on 1 April 2013) Colindale laboratory. Self-sampling was operational from 28 May 2009 through to 18 March 2010 (week 22 2009 to week 112010 ) through either NHSD (28 May to 2 August 2009 and 18 February to 18 March 2010) or NPFS ( 3 August 2009 to 12 February 2010). Data presented here were from 6 August to 18 November 2009, when self-sampling through NPFS was operational for those aged $\geq 16$ years.

\section{Clinician-based sampling through sentinel general practitioners}

Sentinel GPs participating in the clinical surveillance network run by the Royal College of General Practitioners (RCGP) Weekly Returns Service (WRS) took a combined nose and throat swab from patients presenting with influenza-like-illness (ILI) [4]. Swabs were placed in VTM and returned to the HPA Colindale laboratory either by post or a hospital courier system (as previously described) [5]. This scheme was operational continuously between October 2008 and June 2010.

\section{Study period}

The clinician- and self-sampling schemes were compared on samples from those aged $\geq 16$ years, returned within the 15 week period between 6 August 2009 and 18 November 2009, inclusive. During this period of time both schemes were fully operational across England, and were analysed in an identical manner at the HPA Colindale laboratory.
Virological testing by reverse transcription real-time polymerase chain reaction

Returned samples from both surveillance schemes were analysed by real-time polymerase chain reaction (PCR) at the HPA Colindale laboratory as described previously [6-9], testing for $\mathrm{A}\left(\mathrm{H}_{1} \mathrm{~N}_{1}\right)$ pdmog as well as seasonal influenza viruses (influenza $A\left(\mathrm{H}_{1} \mathrm{~N}_{1}\right), A\left(\mathrm{H}_{3} \mathrm{~N}_{2}\right)$ and influenza B). Positive results from all schemes were released to GPs who arranged further clinical management as appropriate.

\section{Statistical analysis}

Linear regression models were used to assess viral shedding post onset of symptoms. Cycle threshold (Ct) values generated by the real-time PCR assays were used as the outcome variable with delay, scheme and age as predictor variables. The $\mathrm{Ct}$ values provided a qualitative positive or negative result that was then used in a logistic regression modelling analysis to compare results obtained from both self-sampling and cliniciansampling schemes. Swabs from the RCGP sentinel virological scheme were taken from patients at the point of presentation to medical services with illness while those taking part in the self-sampling scheme had an inevitable delay due to the posting of the sampling kit to the patient. Any swab obtained where the sampling date was seven or more days post-symptom onset was excluded, as were any swabs for which either the date of swabbing or the date of symptom onset was not recorded. Only adults aged $\geq 16$ years were eligible for self-sampling during the study period, thus data from children were excluded from the RCGP dataset in order to derive an accurate assessment of comparability between the schemes.

The results from the remaining swabs were included in a mixed-effects logistic regression model where the outcome was the binary variable of whether the swab was positive for $A\left(\mathrm{H}_{1} \mathrm{~N}_{1}\right)$ pdmog virus. The following variables were included in the model as fixed effects; centred sequential week number (week), age group $(16-24,25-44,45-64, \geq 65$ years), scheme (selfvs clinician-sampling), and delay (day between symptom onset and swabbing). A composite factor for week and English region (London, West Midlands, North, and South) was included as a random effect to enable the temporal trends to vary between regions. The distribution of the random effects was assumed to be Gaussian. The models were fitted using the xtmelogit command in Stata 11, which utilises adaptive Gaussian quadrature to approximate the log-likelihood.

\section{Ethical approval}

Self-sampling was undertaken as part of a public health surveillance programme in response to the 2009 influenza pandemic and was carried out under NHS Act 2006 (section 251) which provides statutory support for disclosure of such data by the NHS, and the processing by the HPA for communicable disease control. As such, no explicit ethical approval was necessary or sought. 


\section{FIGURE 1}

Comparison of cycle threshold $(\mathrm{Ct})$ values between $\mathrm{A}$ ) self-sampled and B) clinician-sampled influenza A(H1N1) pdm09 polymerase chain reaction positive swabs in adults $\geq 16$ years

A

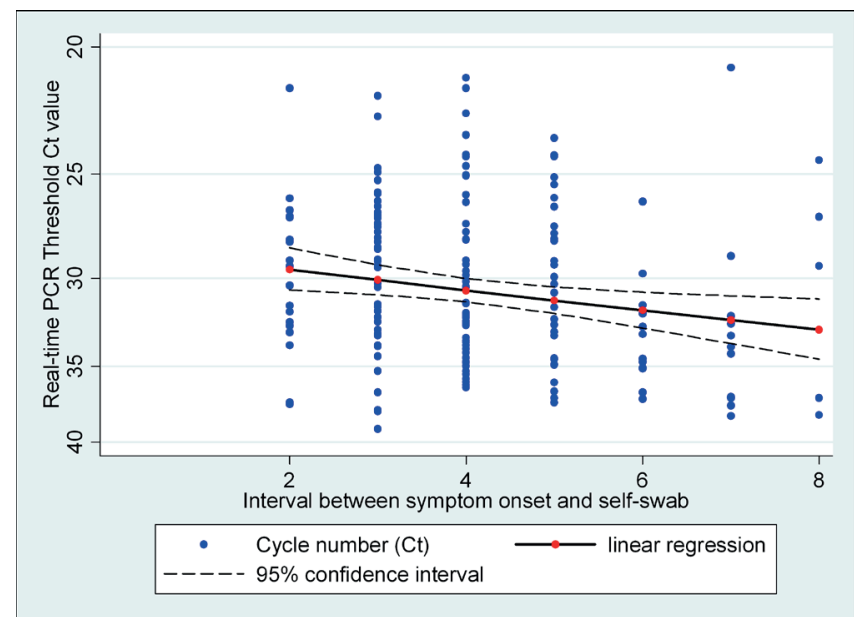

B

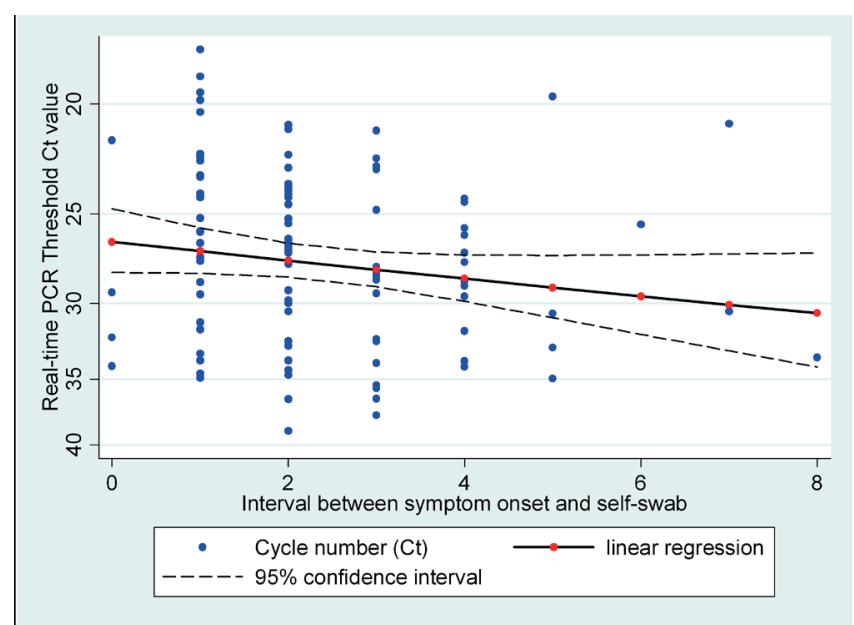

PCR: polymerase chain reaction.

\section{Results}

A total of 26,237 swabs (20,722 self-sampled, and 5,515 clinician-sampled) were received during the pandemic between 28 May 2009 and 18 March 2010, of which 9,292 and 1,949 were within the 15 week study period, respectively. Exclusions included: swabs taken outside England; taken seven or more days after symptom onset; unknown date of swabbing, or symptom onset; contaminated sample (e.g. bacterial or fungal contamination) therefore unsuitable for testing; missing antiviral information (total exclusions: 3,249 (35.0\%) self-sampled, and 803 (41.2\%) GP-sampled swabs). The remaining swabs $(6,043$ self-sampled and 1,146 GP-sampled) were analysed as part of this study with a similar PCR positivity rate for influenza $A\left(\mathrm{H}_{1} \mathrm{~N}_{1}\right)$ pdmo9 of $19.3 \%$, and $25.9 \%$ respectively. To compare the submitted samples from self-sampling and clinician-based sampling, trend analysis of viral load was carried out through analysis of the semiquantitative $\mathrm{Ct}$ values obtained from PCR positive samples collected through both schemes (Figure 1).

There was no evidence of a difference in $\mathrm{Ct}$ values between clinician-based sampling and community self-sampling $(p=0.93)$. There was also no difference between the schemes after the addition of age as a continuous variable for community-based self-sampling $(p=0.15)$ or for clinician-based sampling $(p=0.20)$. Age was also looked at as a categorical variable and there was no impact on the overall model (self-sampling $p=0.45$; clinician sampling $p=0.38$ ). The models were also not affected by adding time/week of swab ( $p=0.26$ for both schemes) nor was there evidence of a regional effect (self-sampling $p=0.32$; clinician-sampling $p=0.067$ ). The results shown in Figure 1 indicate that when comparable samples were analysed there was no significant difference in $\mathrm{Ct}$ values obtained between self-sampling and clinician-led samples.

The sampling of individuals through the community self-sampling scheme was, however, invariably subject to greater delay post-illness onset, because of the time taken for swab kits to be delivered to the patient. There was a clear difference in the delay between onset of symptoms and swabbing in the two schemes, with around half the swabs in the clinician-led scheme taken within two days of symptom onset compared with the four days in the self-sampling scheme (Figure 2). Increasing time post-illness onset is known to correlate with reduced virus shedding in both seasonal and pandemic influenza $[8,10]$.

A mixed-effects logistic regression model incorporating the swabbing results from both schemes was fitted. Differences between PCR positivity over time in the schemes were explored by incorporating an interaction

\section{FIGURE 2}

Empirical cumulative distribution function for the delay between onset of symptoms and date of swabbing in self-sampling ( $\mathrm{n}=6,043$ samples) and clinician-sampling ( $\mathrm{n}=1,146$ samples) schemes

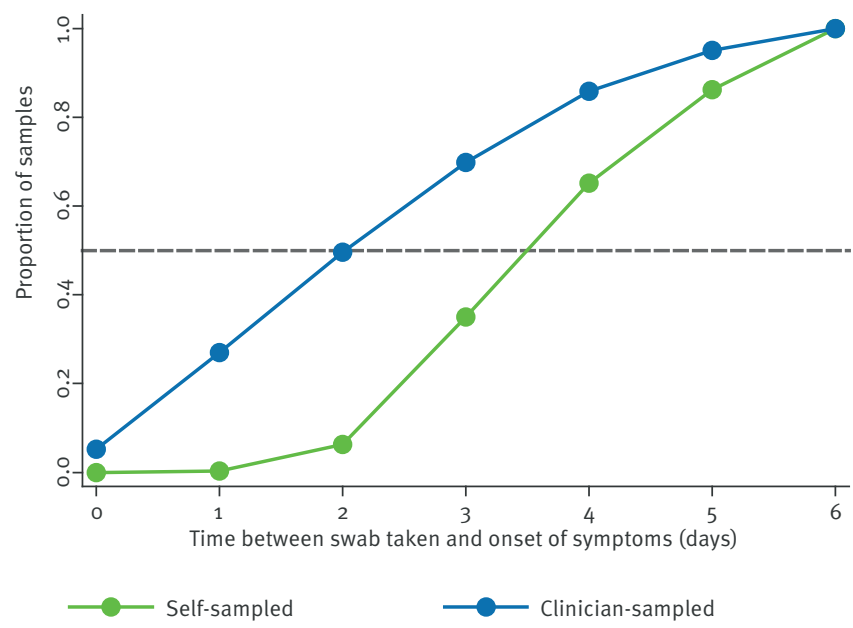




\section{FIGURE 3}

Estimated positivity for influenza A(H1N1)pdm09 from the mixed effects regression model restricted to swabs from 16 to 24 year-olds collected three days post-symptom onset, Northern Strategic Health Authority grouping, England, 2009

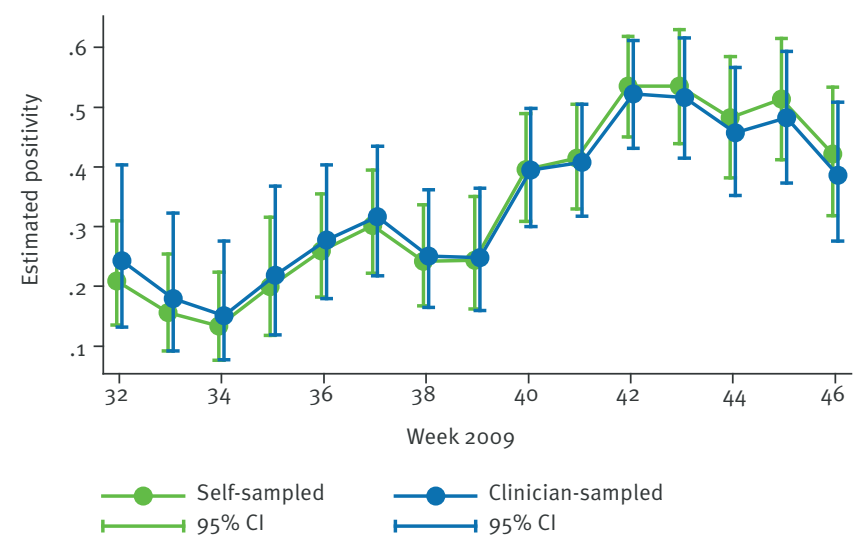

$\mathrm{Cl}$ : confidence interval.

term into the regression model. There was no evidence that the association between positivity and the age group differed between the two schemes, $p=0.3$ (Wald test statistic for interaction $4.89,4$ degrees of freedom (df)). After allowing for the delay in swabbing and age groups there was no evidence that positivity differed systematically between the schemes over time, $p=0.23$ (Wald test statistic for interaction 1.39, $1 \mathrm{df}$ ). Representative results of the regression model are presented in Figure 3 for the 16 to 24 year-old age group, and the northern SHA grouping (North East, North West, Yorkshire and the Humber, and East Midlands) and a three day delay in swabbing (Figure 3). Other age groups and regions exhibited the same temporal differences between the schemes (data not shown).

\section{Discussion}

During the early stages of the pandemic, diagnosis and national level statistics were obtained through accumulation of individual case based diagnostic testing in returning travellers [1]. The transition from individual case based laboratory diagnosis and contact prophylaxis in the initial 'containment phase' to the clinical assessment 'treatment phase' of later stages of the pandemic was managed by using sentinel primary care linked clinical virological surveillance to provide estimates of case numbers. Such monitoring is normally used during the winter months and provides gold standards of surveillance with an extensive historical dataset allowing comparative analysis, but limited predictions [4].

Our work here sought to compare virological surveillance data generated through a self-sampling approach with our gold standard surveillance data generated through clinician led sentinel swabbing practices. The application of this approach during the pandemic supplemented available knowledge in real time [11] and was used for periods of time in parallel with existing RCGP virological surveillance activities.

Self-sampling for near-real time virological surveillance has not been used previously in large scale assessment of respiratory illness in the community during an influenza pandemic. The deployment of this capability was based on our successful pilot scheme in 2008 [2] and was an innovation within the UK health service sector during the global pandemic of 2009/10 [3]. Surveillance was secondary to the provision of patient care, therefore the use of clinical data already available in the health system provided a unique opportunity to evaluate the applicability of the self-sampling approach and assess whether to embed this capability as part of any future national level emergency response strategy.

Self-sampling and clinician-based sampling both provided meaningful semiquantitative data. The analysis of $\mathrm{Ct}$ values demonstrated no difference in viral load trends between the schemes when corrections were made for differences in the timing of swabbing and the delays in sampling. This is encouraging as it suggests significant value in obtaining swabs from patients, to test to influenza, later during infection, at a time when it is usually considered that there is limited opportunity to obtain virological information, thus further improving the potential to use self-sampling where delays in obtaining samples might occur.

The results from this work illustrate that self-sampling for virological surveillance can be used to supplement sentinel clinician-based sampling.

There is a general increasing trend towards self-sampling for illness surveillance, to reduce healthcare costs but also deliver innovative surveillance and screening programmes e.g. the use of self-sampling faecal occult bloods largely for detection of colonic cancer, selfsampling of urines for Chlamydia screening in young persons, and now respiratory illness (both viral and bacterial) sampling [12-18]. These methods may be particularly useful for responding to future influenza epidemics or pandemics which affect younger populations, however, as elderly patients are less likely to use internet or phone health services and therefore may not be included in a self-sampling cohort, this method might be less useful when responding to influenza subtypes that impact on older age groups. In addition, the resources required to develop the infrastructure needed to facilitate the systematic collection of patient data (including: the assembly and dissemination of sampling kits; the collation, analysis and interpretation of data; and the laboratory capacity to test samples) potentially limits the usefulness of self-sampling for responding to small scale or short-lived incidents. However, it may be advantageous to establish 
self-sampling running on a continuous basis, at a low background level, which could contribute to national seasonal influenza surveillance programmes, but also be scaled up to rapidly response to an emerging threat.

It was difficult to get the evidence for community transmission in those going to GPs for testing as they were predominantly in risk groups at the early stages (e.g. returning travellers). Therefore it was hard to objectively assess the necessity for a change in response phase to the pandemic when only a few areas were particularly affected against a background of relatively low numbers of cases in the general population. In aiming to complement sentinel surveillance, our work here clearly demonstrates that respiratory self-sampling can be applied in a responsive and flexible manner to achieve a wide spread and geographically representative way of assessing community transmission of a known organism. Such a scheme could also be invaluable for targeting specific populations in response to public health threats during specific events.

\section{Acknowledgements}

This article is dedicated to Dr Alison Bermingham, who sadly passed away shortly after this manuscript was accepted for publication. Her expertise and contribution in this field will be dearly missed. We are grateful for the work and support from many members of staff from PHE Real-time Syndromic Surveillance Team (Birmingham), and PHE Colindale, London, but in particular Renata Piorkowska and other members of the Respiratory Virus Unit, Virology Specimen Accessions Unit, High-Throughput Molecular Unit, and Respiratory Epidemiology Department. We thank the participating patients and staff from NHS Direct, the National Pandemic Flu Service, the RCGP Weekly Returns Service scheme and Specialist Microbiology Network.

\section{Conflict of interest}

DM Fleming has received funding to attend influenza related meetings and has received consultancy fees from influenza vaccine manufacturers. All other authors: none to declare.

\section{Authors' contributions}

All authors contributed to the design of the study. AJE, CP, DF, EP, GES all contributed to the extraction and processing of NHS Direct call data and the selection of self-sampling participants. AB, JE, CS, PS, AL, DB, MZ contributed to the laboratory testing of samples. $A C$ and $C P$ contributed to the statistical analysis. $A B$ and AJE collaborated to write the manuscript. All authors contributed to drafting and have seen and approved the final version of the manuscript.

\section{References}

1. McLean E, Pebody RG, Campbell C, Chamberland M, Hawkins C, Nguyen-Van-Tam JS, et al. Pandemic (H1N1) 2009 influenza in the UK: clinical and epidemiological findings from the first few hundred (FF100) cases. Epidemiol Infect. 2010;138(11):153141. http://dx.doi.org/10.1017/So950268810001366 PMID:20594381

2. Cooper DL, Smith GE, Chinemana F, Joseph C, Loveridge P, Sebastionpillai $P$, et al. Linking syndromic surveillance with virological self-sampling. Epidemiol Infect. 2008;136(2):222-4.
http://dx.doi.org/10.1017/So950268807008412 PMID:17394678

3. Elliot AJ, Powers C, Thornton A, Obi C, Hill C, Simms I, et al. Monitoring the emergence of community transmission of influenza A/H1 $\mathrm{N}_{1} 2009$ in England: a cross sectional opportunistic survey of self sampled telephone callers to NHS Direct. BMJ. 2009;339(aug27 2):b3403. http://dx.doi. org/10.1136/bmj.b3403 PMID:19713236

4. Ellis JS, Fleming DM, Zambon MC. Multiplex reverse transcription-PCR for surveillance of influenza $A$ and $B$ viruses in England and Wales in 1995 and 1996. J Clin Microbiol. 1997;35(8):2076-82. PMID:9230385

5. Fleming DM, Andrews NJ, Ellis JS, Bermingham A, Sebastianpillai $P$, Elliot AJ, et al. Estimating influenza vaccine effectiveness using routinely collected laboratory data. I Epidemiol Community Health. 2010;64(12):1062-7. http:// dx.doi.org/10.1136/jech.2009.093450 PMID:19910645

6. Ellis J, Iturriza M, Allen R, Bermingham A, Brown K, Gray J, et al. Evaluation of four real-time PCR assays for detection of influenza $A\left(\mathrm{H}_{1} \mathrm{~N}_{1}\right)$ v viruses. Euro Surveill. 2009;14(22):pii=19230. PMID:19497254

7. Ellis JS, Curran MD. Simultaneous molecular detection and confirmation of influenza $\mathrm{AH}_{5}$, with internal control. Methods Mol Biol. 2011;665:161-81. http://dx.doi.org/10.1007/978-160761-817-1_10 PMID:21116801

8. Stephenson I, Democratis J, Lackenby A, McNally T, Smith $J$, Pareek $M$, et al. Neuraminidase inhibitor resistance after oseltamivir treatment of acute influenza $A$ and $B$ in children. Clin Infect Dis. 2009;48(4):389-96. http://dx.doi. org/10.1086/596311 PMID:19133796

9. Wang K, Chalker V, Bermingham A, Harrison T, Mant D, Harnden A. Mycoplasma pneumoniae and respiratory virus infections in children with persistent cough in England: a retrospective analysis. Pediatr Infect Dis J. 2011;30(12):104751. http://dx.doi.org/10.1097/INF.obo13e31822db5e2 PMID:21857262

10. De Serres G, Rouleau I, Hamelin ME, Quach C, Skowronski D, Flamand L, et al. Contagious period for pandemic ( $\left.\mathrm{H}_{1} \mathrm{~N}_{1}\right) 2009$ Emerg Infect Dis. 2010;16(5):783-8. http://dx.doi.org/10.3201/ eid1605.091894 PMID:20409367

11. Health Protection Agency. HPA Weekly National Influenza Report 19 November 2009 (week 47). [Accessed 27 Feb 2015]. Available from: http://webarchive.nationalarchives gov.uk/20140714084352/http://www.hpa.org.uk/web/ HPAweb\&HPAwebStandard/HPAweb_C/1244442494458

12. Akmatov MK, Gatzemeier A, Schughart K, Pessler F. Equivalence of self- and staff-collected nasal swabs for the detection of viral respiratory pathogens. PLOS ONE. 2012;7(11):e48508. http://dx.doi.org/10.1371/journal. pone.0048508 PMID:23155387

13. Akmatov MK, Krebs S, Preusse M, Gatzemeier A, Frischmann $\mathrm{U}$, Schughart K, et al. E-mail-based symptomatic surveillance combined with self-collection of nasal swabs: a new tool for acute respiratory infection epidemiology. Int J Infect Dis. 2011;15(11):e799-803. http://dx.doi.org/10.1016/j. ijid.2011.07.005 PMID:21852171

14. Chai SJ, Aumakhan B, Barnes M, Jett-Goheen M, Quinn $\mathrm{N}$, Agreda $\mathrm{P}$, et al. Internet-based screening for sexually transmitted infections to reach nonclinic populations in the community: risk factors for infection in men. Sex Transm Dis. 2010;37(12):756-63. http://dx.doi.org/10.1097/ OLQ.obo13e3181e3d771 PMID:20644498

15. Coughtrie AL, Whittaker RN, Begum N, Anderson R, Tuck A, Faust SN, et al. Evaluation of swabbing methods for estimating the prevalence of bacterial carriage in the upper respiratory tract: a cross sectional study. BMJ Open. 2014;4(10):e005341. http://dx.doi.org/10.1136/bmjopen-2014-005341 PMID:25358677

16. Garland SM, Tabrizi SN. Diagnosis of sexually transmitted infections (STI) using self-collected non-invasive specimens. Sex Health. 2004;1(2):121-6. http://dx.doi.org/10.1071/ SH03014 PMID:16334994

17. Ip DK, Schutten M, Fang VJ, Fung RO, Dutkowski RT, Chan KH, et al. Validation of self-swab for virologic confirmation of influenza virus infections in a community setting. J Infect Dis. 2012;205(4):631-4. http://dx.doi.org/10.1093/infdis/jir803 PMID:22198963

18. O'Sullivan I, Orbell S. Self-sampling in screening to reduce mortality from colorectal cancer: a qualitative exploration of the decision to complete a faecal occult blood test (FOBT). Med Screen. 2004;11(1):16-22. PMID:15006109 